\title{
A statistical model of fracture for a 2D hexagonal mesh: the Cell Network Model of Fracture for the bamboo Guadua angustifolia
}

\author{
Gabriel Villalobos, José D. Muñoz \\ CeiBA - Complejidad, and Simulation of Physical Systems Group, Department of Physics, \\ Universidad Nacional de Colombia- Bogotá, Crr 30 \# 45-03, Ed. 404, Of. 348, Bogota, Colombia \\ e-mail: gvillalobosc@unal.edu.co \\ Dorian L. Linero \\ Analysis, Design and Materials Group, Department of Civil Engineering, \\ Universidad Nacional de Colombia Bogotá, Crr 30 \# 45-03, Ed. 406 IEI, Of.301
}

July 7,2021

\begin{abstract}
A 2D, hexagonal in geometry, statistical model of fracture is proposed. The model is based on the drying fracture process of the bamboo Guadua angustifolia. A network of flexible cells are joined by brittle junctures of fixed Young moduli that break at a certain thresholds in tensile force. The system is solved by means of the Finite Element Method (FEM). The distribution of avalanche breakings exhibits a power law with exponent -2.93(9), in agreement with the random fuse model [BC06].

Keywords: Statistical models of fracture, Finite Element Method, Computational mechanics of solids. PACS: 02.50.-r, 05.90.+m, 46.50.+a, 62.20.F-, 62.20.M-
\end{abstract}

\section{Introduction}

Guadua angustifolia is an Andean Bamboo that is widely used both as a construction material, for structural frames and walls (TR09]), and as raw material for handicrafts, furniture and vessels, throughout Colombia (Jan81, AV93]). Moreover, Guadua forests play a crucial ecological role, fostering native species, helping to regulate the water cycle, consuming $\mathrm{CO}_{2}$, and injecting water into the soil. Nowadays, it is having a revival as construction material for building houses, $\mathrm{LVV}^{+} 03$ due to recent advances in research about bamboo structural properties and behavior LEM09 as well as structural laminated bamboo $\left[\mathrm{XSC}^{+} 09\right]$.

Guadua structure is optimized to withhold stresses along its axis, with a high Young modulus in this direction, and to bend elastically for deformations perpendicular to it, as all bamboos do. To achieve this performance, all fibers are oriented along the culm axis, more of them being nearer the outer radius than the inner one [NSWP06. Thus, cracks appear mostly along the axis as a consequence of the drying process, due to the avalanche of parenchyma cells collapse.

The statistical models of fracture (SMF) describe macroscopical cracks by following the failure of individual elements ANZ06. An SMF can be tracked by means of computer simulations, which can exhaustively explore the statistical properties of the model. For instance, fiber bundle models are 1D analytically solvable SMFs describing the degradation and failure of materials. (Pei26, Dan45, Sor89, [KRHH06, PHCed]). Furthermore, fracture models have also used to investigate the fractal nature of the cracks MP84, Chi00, the size distribution on impact fragmentation [KH96 and the hydraulic fracture as used on the oil industry GTMC07. All these models consist on individual elements interacting by some known force with their neighbors, where the breaking of a single element redistributes the load on the surrounding ones, causing an avalanche of breaks. Many of them exhibit a phase transition that shows itself in a power-law distribution of these avalanches [KH96, HKPK08, KMHH03, KRHH06]. On the other side, the finite element method has been successfully implemented in the field of computational failure mechanics to study discontinuities and failure dB01, Jir00, $\mathrm{OHP}^{+} 04$.

In the present work we propose a $2 \mathrm{D}$ statistical model of fracture, inspired in the geometry and the mechanical processes of fracture of an Andean Bamboo, Guadua angustifolia. This model is explained in section 2 and resembles the geometry of the $d r y$

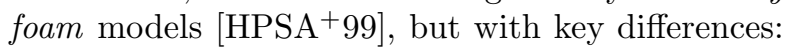


the rule for breaking a juncture here is not random but chosen from the internal forces on the system, the individual hexagonal cells do not share walls and there is no coalescence of neighbouring cells. As in other SMF models, the breaking of a single juncture redistributes the stress on the surrounding ones; but here it is done not by following a simple rule but by computing the new equilibrium state of the whole system. Actually, we use the Finite Element Method (FEM) to model the cell walls and junctures and compute such equilibrium state. All cells are similar in shape, but breaking thresholds are chosen at random. We explore the distribution of avalanches and found that this model exhibits a power-law behaviour (an indication of a phase transition), as other SMF models do.

\section{Guadua structure Model}

Bamboo culms have two main structures: flexible parenchymatous tissue and stiff fiber bundles surrounding the transport vessels (Figure 2). From a structural point of view, the former provide flexibility while the latter carry most of the weight of the plant. During industrial drying, a careful process has to be set up in order to reduce the occurrence of macroscopical cracks that emerge from microcracks at the parenchymatous tissue between the fibers MA06.

Our model, from now on the Cell Network Model of Fracture (CNMF), is a 2D version of the mechanical response of the parenchymatous tissue to the stresses produced by the final drying stage, when all intracellular water is gone. The CNMF is a statistical model of fracture, following the breaking of individual elements. In the real bamboo, cells are joined by the so-called tricellular junctures (made of pectic polysaccharides and calcium [WB07, JM00]), i. e. three individual polymer bonds connecting the cell walls by pairs. The model resembles this structure with uniform hexagonal cells, with six cell walls each, ordered in a honeycomb structure and connected at the corners by triangles of individual junctures.

The model is solved by the Finite Element Method (FEM). Both cell walls and junctures are modeled as linear elastic elements, called Plane Frame Elements (PFE) by the FEM literature Seg84, Bat96, Hug00. Each PFEs has six degrees of freedom: two translational ones and a rotational one at each of two nodes. They are characterized by its elastic modulus $E$, cross sectional area $A$, area moment $I$, and length $L$. The nodal displacements of an element spaning from node $i$ to node $j$ are given by $\overrightarrow{u_{l o c}^{(e)}}=\left\{u_{i}, v_{i}, \phi_{i}, u_{j}, v_{j}, \phi_{j}\right\}$ (Figure 2). In element (or local) coordinates, the element stiffness matrix ( Seg84) is given by:

$\mathbb{K}_{l o c}^{(e)}=\frac{E I}{L^{3}}\left[\begin{array}{cccccc}\frac{A L^{2}}{I} & 0 & 0 & \frac{-A L^{2}}{I} & 0 & 0 \\ 0 & 12 & 6 L & 0 & -12 & 6 L \\ 0 & 6 L & 4 L^{2} & 0 & -6 L & 2 L^{2} \\ \frac{-A L^{2}}{I} & 0 & 0 & \frac{A L^{2}}{I} & 0 & 0 \\ 0 & -12 & 16 L & 0 & 12 & 16 L \\ 0 & 6 L & 2 L^{2} & 0 & -6 L & 4 L^{2}\end{array}\right]$

The representation of this matrix in global coordinates is reached through the transformation

$\mathbb{T}^{(e)}=\left[\begin{array}{cccccc}\cos (\theta) & \sin (\theta) & 0 & 0 & 0 & 0 \\ -\sin (\theta) & \cos (\theta) & 0 & 0 & 0 & 0 \\ 0 & 0 & 1 & 0 & 0 & 0 \\ 0 & 0 & 0 & \cos (\theta) & \sin (\theta) & 0 \\ 0 & 0 & 0 & -\sin (\theta) & \cos (\theta) & 0 \\ 0 & 0 & 0 & 0 & 0 & 1\end{array}\right]$

as $\mathbb{K}^{(e)}=\left(\mathbb{T}^{(e)}\right)^{T} \mathbb{K}_{l o c}^{(e)} \mathbb{T}^{(e)}$. The equilibrium conditions for the whole structure are expressed by

$$
\mathbb{K} \cdot \vec{u}=\overrightarrow{f_{n}}
$$

where $\vec{u}, \mathbb{K}$ and $\vec{f}_{n}$ are the displacement vector, the stiffness matrix and the nodal force vector for the whole structure, respectively, obtained by assembling all individual elements [Seg84]. This expression allows us to compute the displacements from the forces, or viceversa.

Disorder is introduced into the system by choosing at random the breaking thresholds of each juncture. The Young modulus is fixed at literature published values YYFZY7. The initial geometry is regular, so the length $L$ and cross sectional area $A$ of all elements are fixed. As parenchymatous cell walls are ductile and tricellular junctures are brittle, only junctures are allowed to break if their strength surpasses a global failure threshold.

\section{Evolution}

In the final drying stage, that is after the fiber saturation point when all intracellular water is gone, guadua wood is usually part of a structure, and the boundary conditions are fixed even by stress or strain. Let us impose that all boundary nodes are fixed in space, i. e. with displacement zero. The drying process is modeled as a tensile nodal force on all elements that is proportional to the humidity change $\Delta h$. This change increases until a first juncture breaks. Then, the forces redistribute among the resting elements, eventually causing an avalanche of sucessive breakings. The simulation 

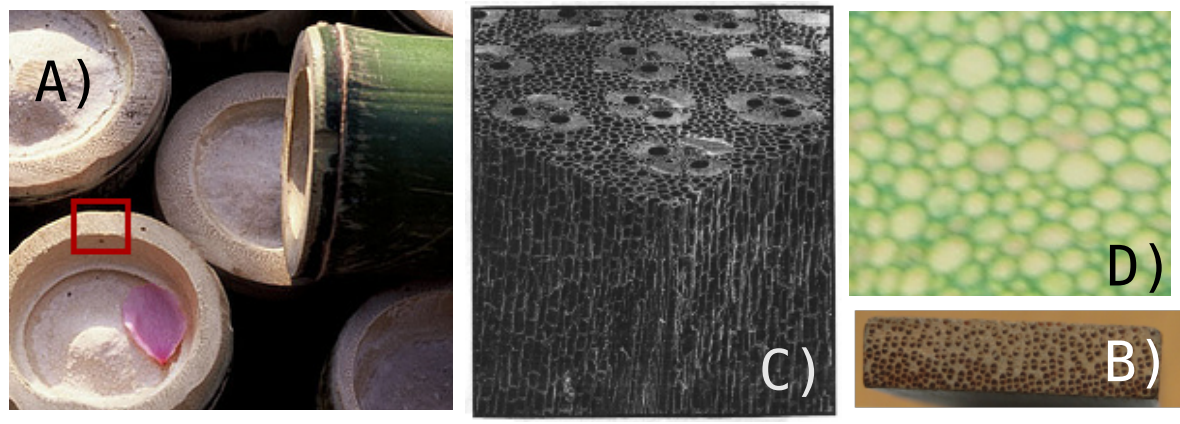

Figure 1: A) Bamboo culms [Lin, B)A small Guadua angustifolia board showing the fibers embedded into the parenchymatous tisse and their distribution: more dense at the outer than at the inner side. C) 3D Bamboo culm structure. Fiber bundles align along the axis of the culm ([Lie98]). D) 2D photography of the Guadua parenchyma $\left(\left[\mathrm{LVV}^{+} 03\right]\right)$.

includes a linear elastic shrink step, a breaking criterium and a non-linear avalanche step.

Linear elastic shrink step: Drying caused stresses are applied following a two step process. In the shrink step, each cell wall suffers shrinking nodal forces along its axis and acting at its ends,

$$
f_{n}^{(\overrightarrow{(e)}}=\left\{-f_{n}, 0,0, f_{n}, 0,0\right\}
$$

with $f_{n}=E A \alpha_{h} \Delta h$. In analogy with the strain caused by temperature WG90, $\alpha_{h}$ is a constant defining how much force is caused by a unit humidity change $\Delta h$. So, increasing $\Delta h$ is like advancing in the drying process. These forces ensembles the global vector $\vec{f}_{n}$. In the equilibrium step, the global displacements $\vec{u}$ are computed from Eq. (3).

Breaking criterium From the global displacement vector $\vec{u}$, the element displacement vectors $\vec{u}^{(e)}$ are constructed. The actual total force $\vec{f}^{(e)}$ acting on a single element due to the contractions and displacements of the whole structure is given by $\vec{f}(e)=\mathbb{K}^{()} \vec{u}^{(e)}$. If the magnitude of the longitudinal component of this vector is larger than the breaking threshold, the element will break. By taking $\Delta h$ as control variable, the secant method gives us the minimum value for a first breaking, that is the drying point when the avalanche starts. At this point, $\Delta h$ is fixed for the whole avalanche and that juncture is broken.

Nonlinear avalanche step. The breaking of a single juncture has two consequences. First, both the global stiffness matrix and the global nodal forces have to be updated. This is achieved by reassembling them from all elements but the broken one, to obtain $\mathbb{K}_{\text {new }}$ and $\vec{f}_{n \text { new }}$. Second, the breaking causes a new global displacement, $\vec{u}_{\text {new }}$, which is computed from Eq. (3). This new displacement may cause in turn a new juncture to break, which causes a new displacement, and so on, until no more junctures break and the avalanche stops.

\section{Results}

Our model consists of an arrangement of 25 cells (Figure 2). The boundary nodes are fixed. Figure 4 shows the number of avalanches as a function of the avalanche size, for different values of the center of the distribution of juncture breaking thresholds. The upper line $(+)$ corresponds to a threshold distribution centered at $10^{-5}$ in units of the area of the cross section of the juncture, $A$, times the mean value of the elastic modulus, $\bar{E}$. The lower line (o) corresponds to a value of 1 in the same units. We averaged over different realizations (this number is also written into the graph). The behaviour of the curves suggests that there is a threshold value where the distribution of avalanches' sizes follows a power law. Our best fit occurs at a threshold of $0.35 A \bar{E}$, with a power law exponent $-2.93(9)$. This value coincides within the error bars with the reported value of 3.0 ([PHH06, HH94]) for the random fuse model ( ometry does not play a mayor role in the breaking statistics.

Acknowledgment: G.V. thanks COLCIEN$C I A S$ for financial support through "Convocatoria Doctorados Nacionales 2008", and Centro de Estudios Interdisciplinarios Básicos y Aplicados en Complejidad-CeiBA - Complejidad for travel financial support. 


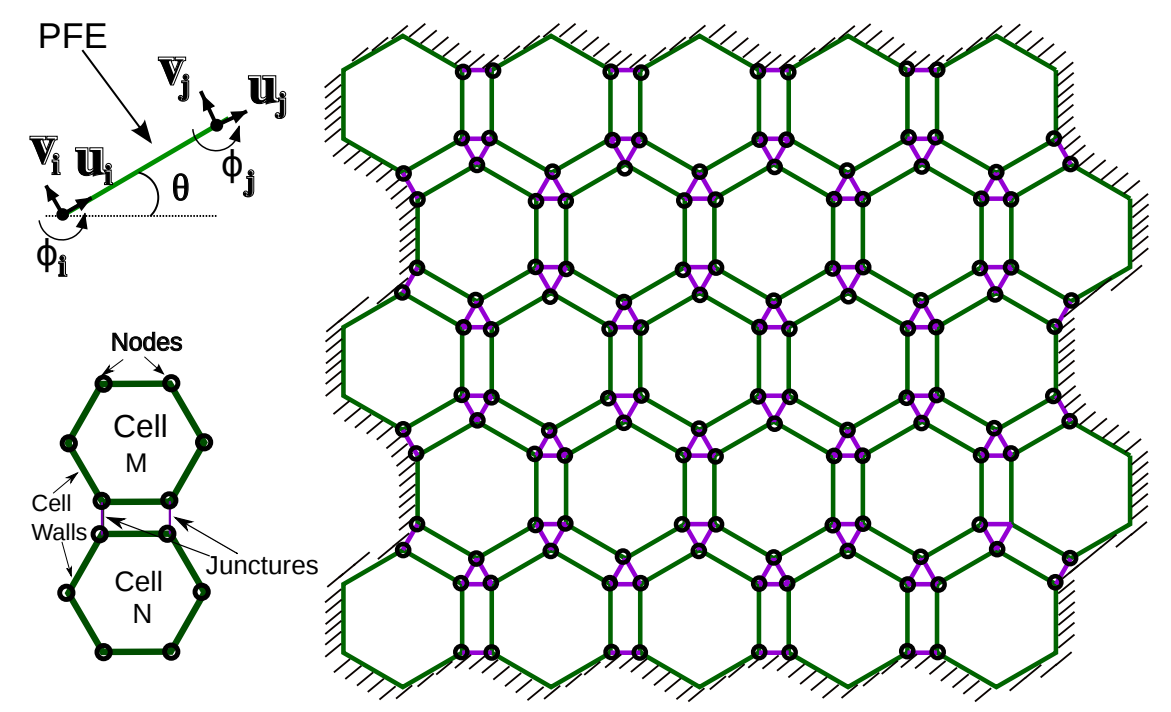

Figure 2: Upper left, the plane frame element spanning between the nodes $i$ and $j$, arbitrarely oriented by an angle $\theta$. Each node has two translational and one rotational degree of freedom. Lower left, two contiguous cells. Right, structure of the CNMF (not at scale). The hexagons represent the cells and the junctures are arranged into triangles. Hashing represents fixed boundary conditions.
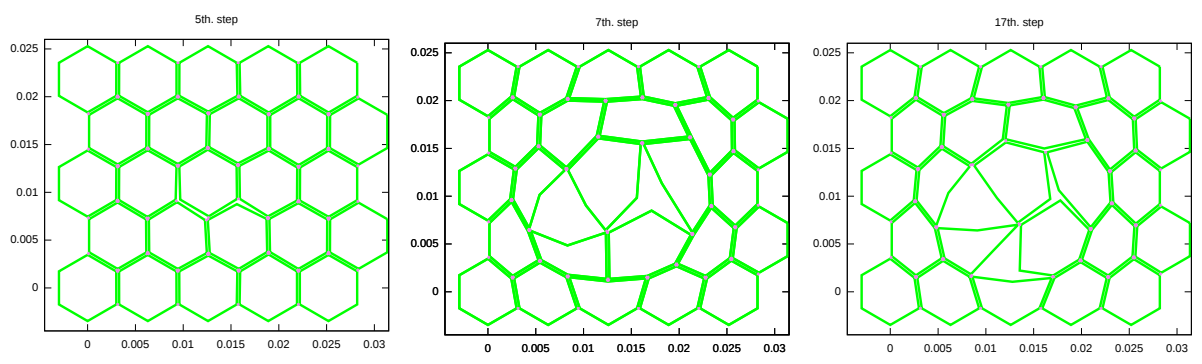

Figure 3: Model evolution. Left(5 steps), a gap appears due to the breaking of some junctures at the center of the sample. Middle, 7 timesteps, right 17 timesteps.

\section{References}

[ANZ06] Mikko J. Alava, Phani K. V. V. Nukala, and Stefano Zapperi. Statistical models of fracture. Advances in Physics, 55:349, 2006.

[AV93] Oscar A. Arce-Villalobos. Fundamentals of the design of Bamboo Structures. PhD thesis, Eindhoven University, 1993. ISBN 90-6814-524-X.

[Bat96] K. Bathe. Finite elements procedures. Prentice Hall, 1996.

[BC06] P. Bhattacharyya and B. Chakrabarti, editors. Modelling critical and catastrophic phemomena in geoscience. Springer, 2006.

[Chi00] Emily S. C. Ching. Multifractality of mass distribution in fragmentation. Physica A: Statistical Mechanics and its Applications, 288(1-4):402 - 408, 2000.

[Dan45] H. E. Daniels. Proc. Roy Soc. London, A183:405, 1945.

[dB01] $\mathrm{R}$ de Borst. Some recent issues in computational failure mechanics. International Journal for Numerical Methods in Engineering, 52:63 - 95, 2001. 


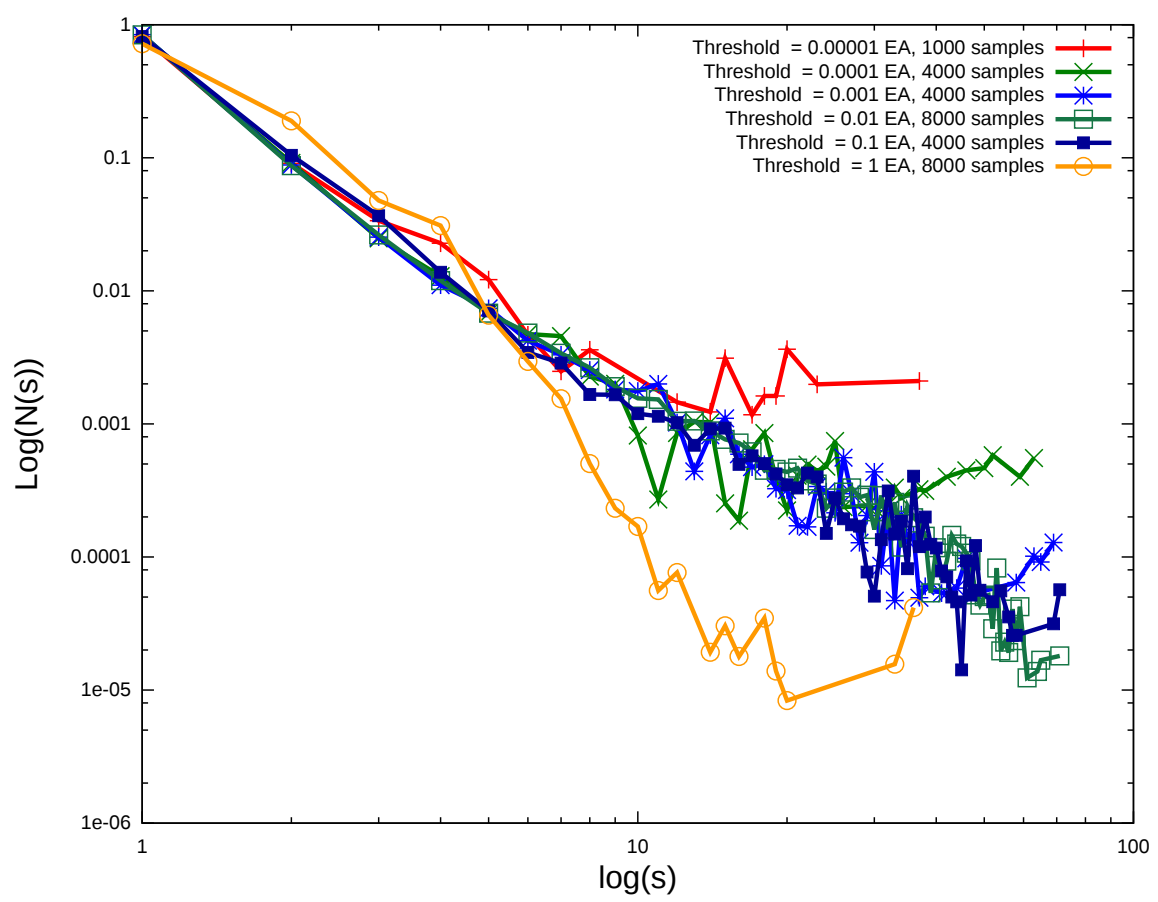

Figure 4: Log-log plot of number of events of avalanche size $s(\log (N(s)))$ as function of avalanche sizes $(\log (s))$ for the CNMF, for several centers of distribution of breaking thresholds. A power law with exponent -2.93(9) is gather for a breaking threshold of 0.35 (for an explanation on the units, see Sec. 4)

[GTMC07] Sergio Andres Galindo Torres and José Daniel Muñoz Castaño. Simulation of the hydraulic fracture process in two dimensions using a discrete element method. Phys. Rev. E, 75(6):066109, Jun 2007.

[Her90] Hans J. Herrmann, editor. Statistical Models for the Fracture of Disordered Media. Random materials and processes. North-Holland, 1990.

[HH94] A. Hansen and P.C. Hemmer. Phys. Lett. A., 184(394), 1994.

[HKPK08] R. C. Hidalgo, K. Kovacs, I. Pagonabarraga, and F. Kun. Universality class of fiber bundles with strong heterogeneities. EPL, 81:54005, 2008.

[HPSA $\left.{ }^{+} 99\right]$ A. Hasmy, R. Paredes, O. Sonneville-Aubrun, B. Cabane, and R. Botet. Dynamical transition in a model for dry foams. Phys. Rev. Lett., 82(16):3368-3371, Apr 1999.

[Hug00] T. J. R. Hughes. The finite element method. Dover, 2000.

[Jan81] Julius J.A. Janssen. Bamboo in building structures. PhD thesis, Eindhoven University, 1981.

[Jir00] M. Jirasek. Comparative study on finite elements with embedded discontinuities. Computer Methods in Applied Mechanics and Engineering, 188:307 - 330, 2000.

[JM00] Michael C. Jarvis and Maureen C. McCann. Macromolecular biophysics of the plant cell wall: Concepts and methodology. Plant Physiology and Biochemistry, 38(1-2):1 - 13, 2000.

[KH96] Ferenc Kun and Hans J. Herrmann. A study of fragmentation processes using a discrete element method. Computer Methods in Applied Mechanics and Engineering, 138(1-4):3 - 18, 1996.

[KMHH03] F. Kun, Y. Moreno, R.C. Hidalgo, and H.J. Herrmann. Creep rupture has two universality classes. Europhys. Lett., 63:347, 2003. 
[KRHH06] F. Kun, F. Raischel, R. C. Hidalgo, and H. J. Herrmann. Modelling Critical and Catastrophic Phenomena in Geoscience, chapter Extensions of fiber bundle models. 2006.

[LEM09] Khosrow Ghavami Luís Eustáquio Moreira. Bamboo space structure. In 11th International Conference on Non-conventional Materials and Technologies NOCMAT, 2009.

[Lie98] Walter Liese. The Anatomy of Bamboo Culms. 1998. ISBN 81-86247-26-2.

[Lin] LinkesAuge. LinkesAuge flickr user.

$\left[\mathrm{LVV}^{+} 03\right]$ X. Londoño, Marcelo Villegas, Simón Velez, Jesús Velez, Gabriel G. Londoño, and Götz Schmitt. Guadua Arquitecutra y Diseño. Villegas Editores, 2003.

[MA06] Jorge Augusto Montoya Arango. Trocknungsverfahren furr die Bambusart Guadua angustifolia unter tropischen Bedingungen. PhD thesis, Universitat Hamburg, 2006.

[MP84] Benoit Mandelbrot and Dann E. Passoia. Fractal character of fracture surfaces of metals. Nature, 308(19), April 1984.

[NSWP06] Emilio Carlos Nelli Silva, Matthew C Walters, and Glaucio H Paulino. Modeling bamboo as a functionally graded material: lessons for the analysis of affordable materials. Journal of Matherial Sciences, 41:6991 - 7004, 2006.

$\left[\mathrm{OHP}^{+}\right.$04] J. Oliver, A. E. Huespe, M. D. G. Pulido, S. Blanco, and D. L. Linero. New developments in computational material failure mechanics. Sixth World Congress on Computational Mechanics (WCCM VI), Beijing, (P.R. of China),, Tshinghua University Press., 2004.

[Pei26] F. T. Peirce. J. Text. Ind., 17:355, 1926.

[PHCed] Srutarshi Pradhan, Alex Hansen, , and Bikas K. Chakrabarti. Failure processes in elastic fiber bundles. Reviews of Modern Physics, 2009 - Accepted.

[PHH06] Srutarshi Pradhan, Alex Hansen, and Per C. Hemmer. Crossover behavior in failure avalanches. Phys. Rev. E, 74(1):016122, Jul 2006.

[Seg84] Larry J. Segerlind. Applied Finite Element Analysis. John Wiley and sons, 1984.

[Sor89] D. Sornette. J. Phys. A, 22:L243, 1989.

[TR09] Caori. Takeuchi and Jhon Fredy. Rivera. Structural behaviour of braced guadua frames. In 11th International Conference on Non-conventional Materials and Technologies NOCMAT, 2009 .

[WB07] Keith W. Waldron and Christopher T. Brett. Plant Cell Separation and Adhesion, chapter The role of polymer cross-linking in intercellular adhesion. Blackwell Publishing, 2007.

[WG90] William Weaver and James M. Gere. Matrix Analysis of Framed Structures. Van Nostrand Reinhold, 3rd. edition, 1990.

$\left[\mathrm{XSC}^{+} 09\right]$ Y. Xiao, B. Shan, G. Chen, Q. Zhou, R.Z Yang, and L.Y. She. Development of laminated bamboo modern structures. In 11th International Conference on Non-conventional Materials and Technologies NOCMAT, 2009.

[YFZY07] Yan Yu, Benhua Fei, Bo Zhang, and Xiang Yu. Cell-wall mechanical properties of bamboo investigated by in-situ imaging nanoindentation. Wood and Fiber Science, 39(4):527 - 535, October 2007. 